\title{
A hybrid qPCR/SNP array approach allows cost efficient assessment of KIR gene copy numbers in large samples
}

\author{
Nikolas Pontikos ${ }^{1}$, Deborah J Smyth ${ }^{1}$, Helen Schuilenburg ${ }^{1}$, Joanna MM Howson ${ }^{1,2}$, Neil M Walker ${ }^{1}$, \\ Oliver S Burren', Hui Guo1, Suna Onengut-Gumuscu³, Wei-Min Chen ${ }^{3}$, Patrick Concannon ${ }^{4}$, \\ Stephen S Rich ${ }^{3}$, Jyothi Jayaraman ${ }^{5,6}$, Wei Jiang 5,6, James A Traherne ${ }^{5,6}$, John Trowsdale 5,6, \\ John A Todd ${ }^{1+}$ and Chris Wallace ${ }^{1,7^{*}+}$
}

\begin{abstract}
Background: Killer Immunoglobulin-like Receptors (KIRs) are surface receptors of natural killer cells that bind to their corresponding Human Leukocyte Antigen (HLA) class I ligands, making them interesting candidate genes for HLA-associated autoimmune diseases, including type 1 diabetes (T1D). However, allelic and copy number variation in the KIR region effectively mask it from standard genome-wide association studies: single nucleotide polymorphism (SNP) probes targeting the region are often discarded by standard genotype callers since they exhibit variable cluster numbers. Quantitative Polymerase Chain Reaction (qPCR) assays address this issue. However, their cost is prohibitive at the sample sizes required for detecting effects typically observed in complex genetic diseases.

Results: We propose a more powerful and cost-effective alternative, which combines signals from SNPs with more than three clusters found in existing datasets, with qPCR on a subset of samples. First, we showed that noise and batch effects in multiplexed QPCR assays are addressed through normalisation and simultaneous copy number calling of multiple genes. Then, we used supervised classification to impute copy numbers of specific KIR genes from SNP signals. We applied this method to assess copy number variation in two KIR genes, KIR3DL1 and KIR3DS1, which are suitable candidates for T1D susceptibility since they encode the only KIR molecules known to bind with HLA-Bw4 epitopes. We find no association between KIR3DL1/3DS1 copy number and T1D in 6744 cases and 5362 controls; a sample size twenty-fold larger than in any previous KIR association study. Due to our sample size, we can exclude odds ratios larger than 1.1 for the common KIR3DL1/3DS1 copy number groups at the 5\% significance level.

Conclusion: We found no evidence of association of KIR3DL1/3DS1 copy number with T1D, either overall or dependent on HLA-Bw4 epitope. Five other KIR genes, KIR2DS4, KIR2DL3, KIR2DL5, KIR2DS5 and KIR2DS1, in high linkage disequilibrium with KIR3DL1 and KIR3DS1, are also unlikely to be significantly associated. Our approach could potentially be applied to other KIR genes to allow cost effective assaying of gene copy number in large samples.
\end{abstract}

Keywords: KIR3DL1, KIR3DS1, KIR2DS4, KIR2DL3, KIR2DL5, KIR2DS5, KIR2DS1, HLA-Bw4, CNV, qPCR, ImmunoChip, KIR, Imputation, T1D

\footnotetext{
*Correspondence: chris.wallace@cimr.cam.ac.uk

${ }^{\dagger}$ Equal contributors

1 JDRF/Wellcome Trust Diabetes and Inflammation Laboratory,

Cambridge Institute for Medical Research, University of Cambridge,

Wellcome Trust/MRC Building, CB2 OXY, Cambridge, UK

7 MRC Biostatistics Unit, Institute of Public Health, University Forvie Site,

Robinson Way, CB2 OSR, Cambridge, UK

Full list of author information is available at the end of the article
} 


\section{Background}

Killer Immunoglobulin-like Receptors (KIRs) are transmembrane glycoproteins expressed by natural killer cells and subsets of $\mathrm{T}$ cells. The KIR region lies in a $150 \mathrm{~kb}$ gene cluster located within the $1 \mathrm{Mb}$ Leukocyte Receptor Complex on chr19q13.4. The region exhibits great haplotype and copy number diversity, which has prevented complete assessment of the KIR genes in standard genome-wide association studies (GWAS), despite their strong candidacy for immune-related traits. Targeted quantitative Polymerase Chain Reaction (qPCR) assays have been used to detect presence or absence of individual KIR genes and more recently, determine copy numbers [1]. Nevertheless these remain expensive and labour intensive compared to SNP arrays.

We show that SNPs often discarded in GWAS, because they exhibit non-typical number of genotype clusters, can be informative of KIR gene copy numbers. By applying supervised classification, we are able to use qPCR results in a modest number of samples to impute copy numbers into a larger sample for which SNP array signals are available. We illustrate this method by applying it to two genes in the KIR complex, KIR3DL1 and KIR3DS1, which are suitable candidates for T1D association due to their interaction with HLA class I molecules. Specifically, the KIR3DL1 protein is known to interact with the HLA class I allotypes that contain the HLA-Bw4 serological epitope $[2,3]$, whereas the protein encoded by KIR3DS1, which shares $97 \%$ sequence similarity to KIR3DL1, is thought to bind the more restrictive HLABw4-80I epitope subset [4]. The grouping of HLA-A and HLA-B alleles according to HLA-Bw4 serological epitope [5] is given in Additional file 1: Table S1 and includes several HLA class I alleles which are associated with T1D risk after conditioning on the major HLA class II effects [6,7]. To date, KIR3DL1/3DS1 association with T1D has only been studied using qPCR assays in limited sample sizes, which assess presence or absence of each KIR gene [8].

We used qPCR copy number calls in 1474 samples as a training set, and imputed copy number in a further 12106 samples from raw genotyping signals in SNP array probes targeting the KIR region. We thus tested association of KIR3DL1/3DS1 copy number with T1D, either directly, or through interaction with HLA-Bw4. To the best of our knowledge, the sample size of our study is twenty-fold larger than any previous study of KIR3DL1/3DS1 in T1D, and the first to test copy number variation rather than simply presence or absence [8].

The hybrid method we advocate, leverages the information available from targeted qPCR assays in modest samples to the level of sample coverage required for modern, well-powered genetic studies. It has the potential to be applied to other genes in the KIR region or, indeed, to other chromosome regions that exhibit similar copy number variation and sequence complexity.

\section{Methods}

\section{Subjects}

DNA was available from 12106 individuals: 6744 cases (age of diagnosis less than 17) from the Genetic Resource Investigating Diabetes (GRID) cohort, and 5362 controls from the British 1958 Birth Cohort (1958BC). All subjects were of white European ancestry with written informed consent and Ethics Committee/Institutional Review Board approval. The GRID cohort was approved by the Cambridgeshire 4 Research Ethics Committee, study title "Developing targets for Diabetes prevention by the study of the genetics of Type 1 diabetes" (ref $00 / 5 / 044)$. The 1958BC cohort was approved by the North West Ethics Committee, study title "1958 Birth Cohort Tissue Bank" (ref 09/H1010/12). The use of these samples was approved by the Cambridgeshire 2 Research Ethics Committee, study title "Investigating Genes and Phenotypes associated with Type 1 Diabetes" (ref 08/H0308/153).

Ancestry was confirmed by PCA analysis of earlier GWAS data in these samples [9]. The DNA for the cases and controls was prepared using the same protocols in Cambridge and in Bristol respectively. All samples were cell-line derived.

HLA genotypes were available on a subset of 5603 individuals, 2922 cases and 2681 controls. HLA-A and HLA-B genes were typed at four-digit allele resolution using Dynal RELI SSO assays (Invitrogen, Paisley, U.K.) (Additional file 1: Table S3). The epitope classification of HLA-A and HLA-B alleles is given in Additional file 1: Table S1. All 12106 samples have been genotyped using ImmunoChip, a custom Illumina 200K Infinium high-density SNP array [10], according to the manufacturer's protocol, processed at the University of Virginia in Charlottesville, USA. A random subset of 1629 samples, 816 cases and 813 controls, for which HLA genotype was available, were selected for qPCR. These samples were arrayed on bespoke 96-well plates, randomised half cases, half controls. The source plates were chosen as those containing samples with the most HLA typing available at the time (2009).

\section{Design of multiplexed qPCR KIR3DL1/3DS1 copy number assay}

The qPCR platform used was the LightCycler 480 RealTime PCR Instrument on which we ran eighteen 384well plates and four repeated plates. The 1629 samples, 816 cases and 813 controls, selected for qPCR were arrayed evenly, half-cases, half-controls, across the plates. On the four repeated plates, 310 samples were arrayed (Additional file 1: Figure S3). On each plate, every sample 
was replicated across four neighbouring wells, resulting in a maximum of 96 samples per plate. All plates, except for one, contained four repeated calibrator samples of known $K I R 3 D L 1 / 3 D S 1$ copy number that included, two samples with KIR3DS1-KIR3DL1 copy number 1-2 and two samples with KIR3DS1-KIR3DL1 copy number 2-1. These are represented in Additional file 1: Figure S2 as black points. To detect contamination, each plate also included one water well. Four plates were analysed in duplicate in order to assess reproducibility (Additional file 1: Figure S3).

The qPCR probes and forward/reverse primers were carefully designed, in collaboration with Jiang et al. [1], to target and amplify most known KIR3DL1 and KIR3DS1 alleles, as well as the reference gene STAT6, known to always be present in two copies. The probe and primer sequences are summarised in Additional file 1: Table S2.

Each qPCR well was multiplexed, so that the copy numbers of KIR3DL1, KIR3DS1 and STAT6 were simultaneously assayed as part of the same qPCR reaction. To allow for this, the probes were conjugated with three distinct dyes: Fam for KIR3DS1, Cy5 for KIR3DL1 and DFO for STAT6.

Each qPCR well reaction was prepared with $2 \mu$ l of DNA at $5 \mathrm{ng} \mu \mathrm{l}^{-1}$ and $5 \mu \mathrm{l}$ of Quantifast Multiplex PCR master$\operatorname{mix}(0.25 \mu \mathrm{l}$ primer mix, $0.045 \mu \mathrm{l}$ probe mix and $4.705 \mu \mathrm{l}$ of water). The qPCR conditions were $95^{\circ} \mathrm{C}$ for $5 \mathrm{~min}$, followed by 40 cycles at $95^{\circ} \mathrm{C}$ for $15 \mathrm{~s}$ and $66^{\circ} \mathrm{C}$ for $50 \mathrm{~s}$. Data was collected at $66^{\circ} \mathrm{C}$.

\section{Quality control and normalisation of the qPCR data}

The experiment files exported from the LightCycler gave us three crossingpoint $(\mathrm{Ct})$ values per well, one for each of the dye-DNA conjugates. The $\mathrm{Ct}$ value is representative of the number of $\mathrm{qPCR}$ cycles required for the dye-DNA conjugate to be sufficiently amplified for the fluorescence to cross the detection threshold. Hence a larger $\mathrm{Ct}$ value usually implies a smaller underlying copy number. For each well, by subtracting the Ct of Fam-KIR3DL1 and Cy5-KIR3DS1 from the Ct value of the DFO-STAT6, the reference dye-DNA conjugate, we obtained the baseline relative $\Delta C t$ value for KIR3DS1 and KIR3DL1. Then for each sample, we took the median over the four replicate wells to obtain per sample, KIR3DS1 and KIR3DL1 $\Delta \mathrm{Ct}$ values (Additional file 1: Figure S1a.b). However, certain wells did not yield a STAT6 Ct value since the detection threshold was not crossed within the 40 PCR cycles. We found 64 samples that did not yield a DFOSTAT6 Ct reading in all four well replicates and these were excluded in the first step of our quality control (QC). Visual inspection of the KIR3DL1 and KIR3DS1 $\triangle \mathrm{Ct}$ distributions by plate led us to drop plate 22 (highlighted in Additional file 1: Figure S1a.b) as it appeared to contain numerous outliers. This resulted in a further 91 samples being dropped as part of our QC. Following QC, we were left with 1474 unique samples, 747 cases and 727 controls, over 17 plates.

Individual distributions of KIR3DS1 and KIR3DL1 $\Delta \mathrm{Ct}$ were not aligned between plates (Additional file 1: Figure S1.c.d), this prevented pooling of all plates for copy number calling. To align the $\Delta \mathrm{Ct}$ distributions across the 17 plates, we first applied the k-medoids algorithm within each plate to the $\triangle \mathrm{Ct} K I R 3 D S 1$ and KIR3DL1 separately to identify the location of the most distinguishable copy number groups, one and two copies. We then normalised across plates by a linear transformation so that the median $\Delta \mathrm{Ct}$ of the two groups mapped to 1 and 2 across all 17 plates (Additional file 1: Figure S1.e.f). After normalisation, negative $\Delta \mathrm{Ct}$ values were assigned to zero to reflect their expected copy number state.

Following QC and normalisation, samples which were repeated across different plates showed good reproducibility (Additional file 1: Figure S3). These were summarised by the median of their repeated value.

\section{Copy number calling and multiple imputation in the subset of samples with qPCR data}

Samples with three or more missing Fam-KIR3DL1 or Cy5-KIR3DS1 Ct values out of the four well replicates, were assigned to zero copies of KIR3DL1 or KIR3DS1 respectively.

For the remainder of the samples, copy number calling was done jointly on KIR3DL1 and KIR3DS1 using unsupervised clustering with a finite mixture model. We called copy number groups by fitting a mixture of eight bivariate Gaussian distributions to exploit the notable correlation between the normalised KIR3DS1 and KIR3DL1 $\triangle \mathrm{Ct}$ values (Additional file 1: Figure S2). We allowed for eight KIR3DS1-KIR3DL1 copy number groups: three common groups of two copy numbers $(0-2,1-1,2-0)$ and five rarer groups of lower or higher copy numbers $(2-1,1$ 2, 0-1, 1-0, 3-0) (Figure 1). The mixture was fitted using an EM algorithm [11] with initial parameters calculated from the clusters returned by k-means with centers set to the eight expected locations of the copy number groups. After fitting the mixture model, each sample was assigned eight posterior probabilities of belonging to each of the eight copy number groups, allowing for uncertainty in copy number calling. These posterior probabilities were used to simulate ten plausible KIR3DS1-KIR3DL1 copy number datasets. These ten multiply imputed datasets allowed for statistical analysis to be conducted in parallel and inference to be combined across datasets using the methods described by Little and Rubin (1987) [12] and implemented in the mitools and mice $\mathrm{R}$ packages $[13,14]$. 


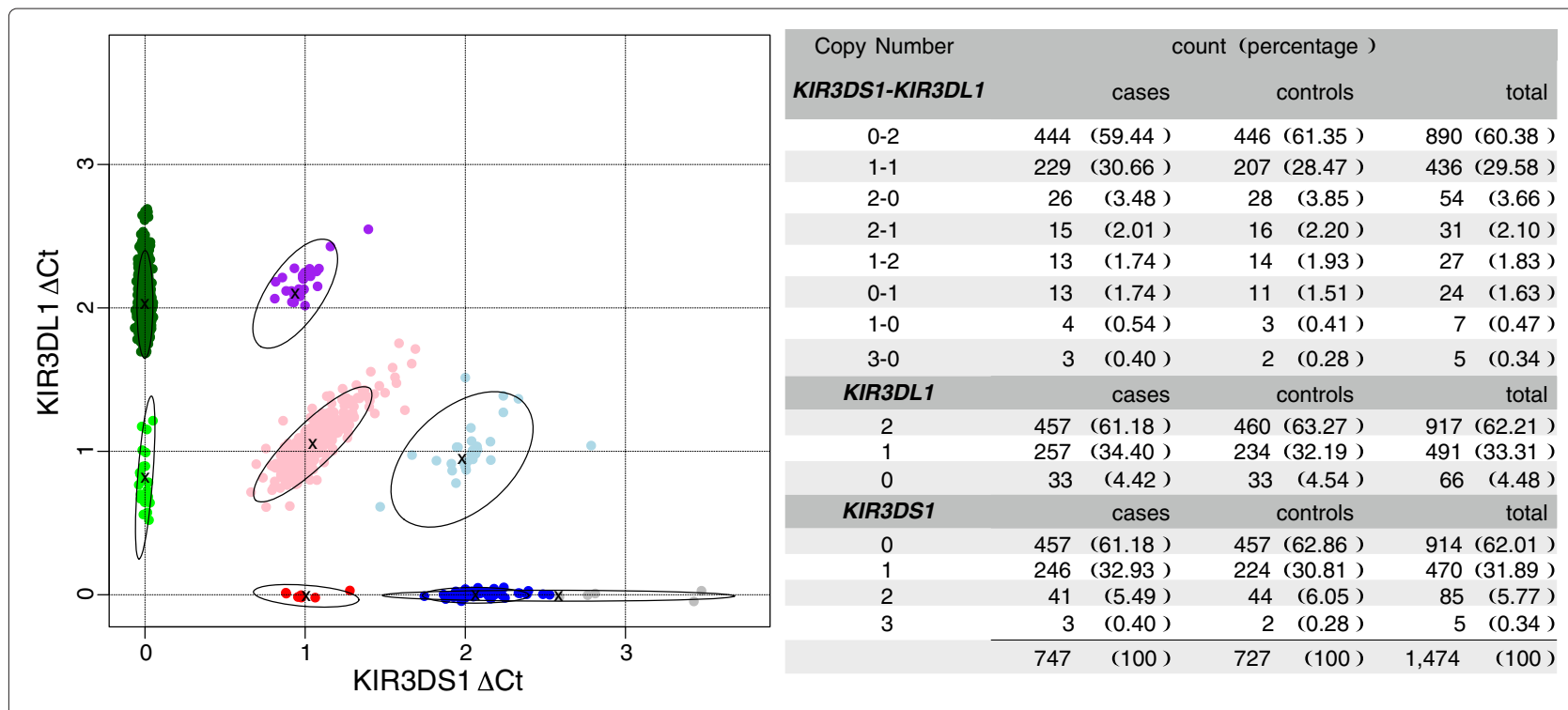

Figure 1 Bivariate copy number calling of KIR3DL1/3DS1 from qPCR $\Delta \mathrm{Ct}$. On the left, the median normalised $\Delta \mathrm{Ct}$ values for KIR3DS1 and KIR3DL1 are shown with the results of clustering into the eight copy number groups coloured according to the group with the highest posterior probability. The three most common KIR3DS1-KIR3DL1 copy number groups are the ones with a total copy number of two: 0-2 (dark green), 1-1 (pink) and 2-0 (dark blue). The ellipses delimit the $95^{\text {th }}$ percentile. On the right, the counts of the most probable copy number groups are shown for cases and controls.

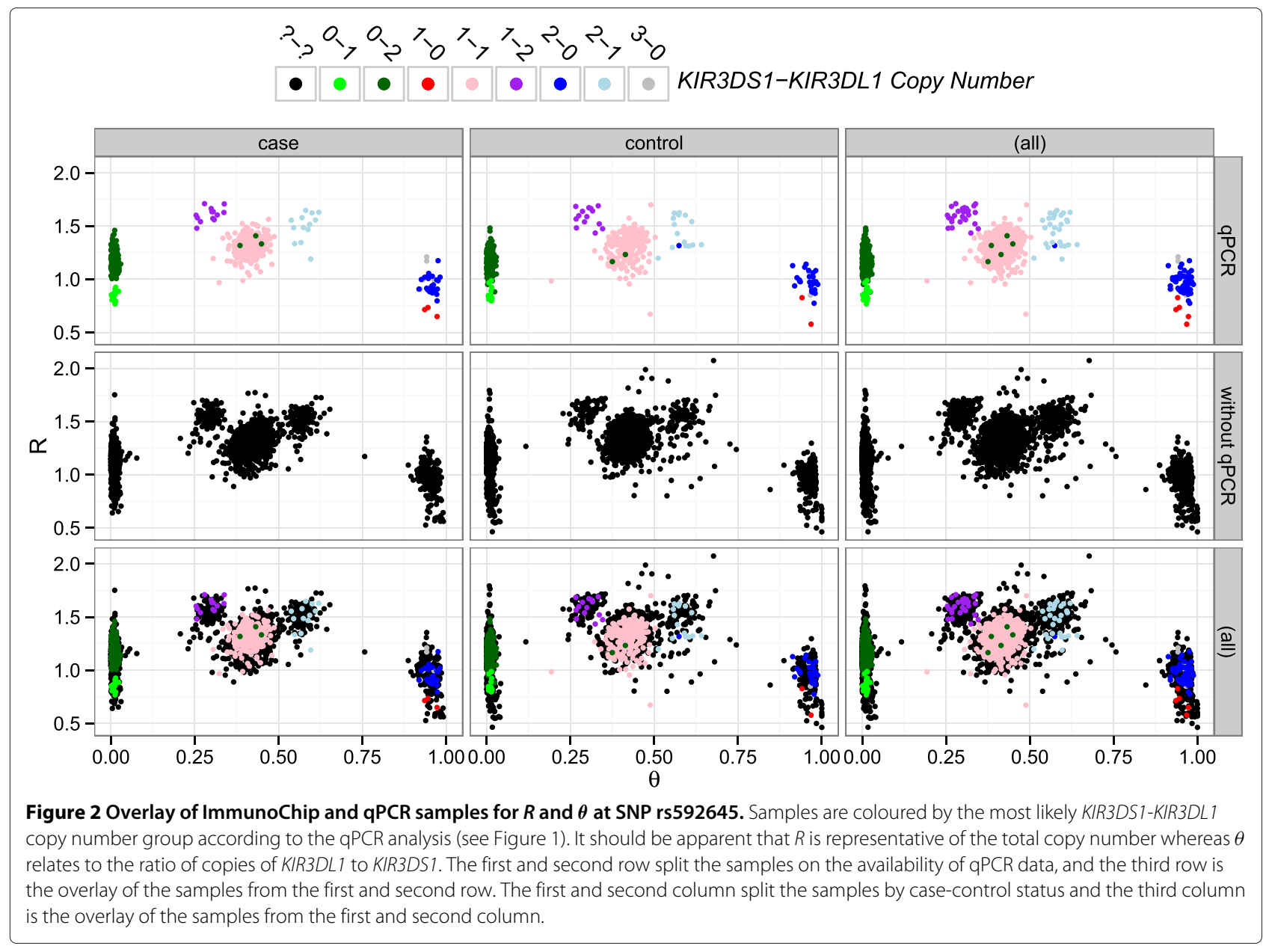




\section{Copy number imputation into the extended samples}

We extended our sample size by using the subset of samples common between the qPCR and SNP datasets, 747 cases and 727 controls, to train a k-nearest neighbour (knn) classifier to predict KIR3DL1/3DS1 copy number using the $R$ and $\theta$ signals from ImmunoChip SNPs.

Illumina arrays, such as the ImmunoChip, have two fluorescent probes which differ on one base and allow discrimination of biallelic SNPs. The fluorescent intensities are $X$ and $Y$, from which are derived the sum, $R=X+Y$, and the ratio, $\tan (\theta)=\frac{X}{Y}$.

Each of 30 SNPs lying within the KIR3DL1/3DS1 region, were assessed for association with either KIR3DL1 or KIR3DS1 copy number in individual linear regression of copy number against $R$ and $\theta$ (Additional file 1: Table S4). Nineteen SNPs out of 30 were associated (p-value < 0.05), nine of which would have failed ImmunoChip QC (Additional file 1: Table S4), with rs592645 the most strongly predictive (Figure 2). We compared running knn with all predictive SNPs or on various subsets, and found rs592645 alone, with $k=8$, minimised the mean leaveone-out cross-validation (LOOCV) error rate over ten multiply imputed qPCR datasets (Figure 3 ). In each multiply imputed qPCR dataset, all samples were assigned a single imputed copy number group. We also explored the effect of varying the size of the training data set by setting KIR gene copy numbers to missing for a randomly chosen subset of samples and imputing them in the remaining samples (Figure 4).

\section{Testing for association of KIR3DL1/3DS1 copy number with T1D}

We tested for association of T1D with the predicted copy numbers from the qPCR and SNP datasets using logistic regression. We allowed for uncertainty in the copy number call when estimating individual odds ratios by using the ten multiply imputed datasets generated from the qPCR posterior probabilities [15], and averaging the estimates over those with the $R$ mitools package [13]. We allowed for statistical interaction with HLA-Bw4 by repeating the association test in the subsets of carriers of the target ligand HLA-Bw4 epitopes, HLA-Bw4 for KIR3DL1 and the putative ligand HLA-Bw4-80I for KIR3DS1. We directly tested for interaction with a more powerful case-only $\chi^{2}$ test $[16,17]$.

\section{Results and discussion}

Bivariate clustering enables accurate copy number calling in $\mathrm{QPCR}$ data

Before normalisation, $\Delta \mathrm{Ct}$ distributions varied across plates preventing simple visual copy number assignment (Additional file 1: Figure S1). After normalisation, samples repeated across different plates showed good reproducibility (Additional file 1: Figure S3). Furthermore, bivariate clustering, on both the KIR3DS1 and KIR3DL1

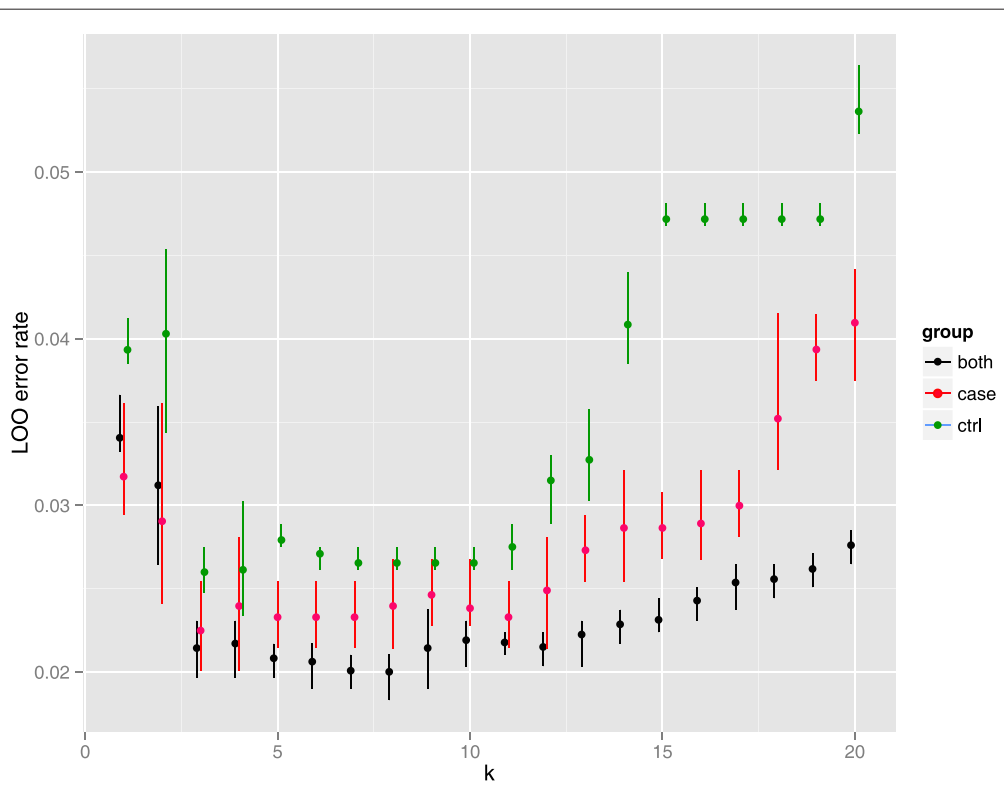

Figure 3 Leave-one-out crossvalidation error rate for k-nearest neighbour prediction. Leave-one-out cross validation error rates obtained from $\mathrm{k}$-nearest neighbours (knn) prediction of KIR3DL1/3DS1 copy numbers from the $R$ and $\theta$ signals of SNP rs592645. Each point shows the proportion of samples for which the knn predicted copy number did not match the qPCR call, averaged over ten multiply imputed qPCR call datasets (using the posterior probabilities from Figure 1). Error bars show the minimum and maximum error rates over the ten multiply imputed datasets. Knn was run in parallel for cases only, controls only and on all samples together. The minimum error rate is achieved for $k=8$ when the prediction uses both cases and controls. 


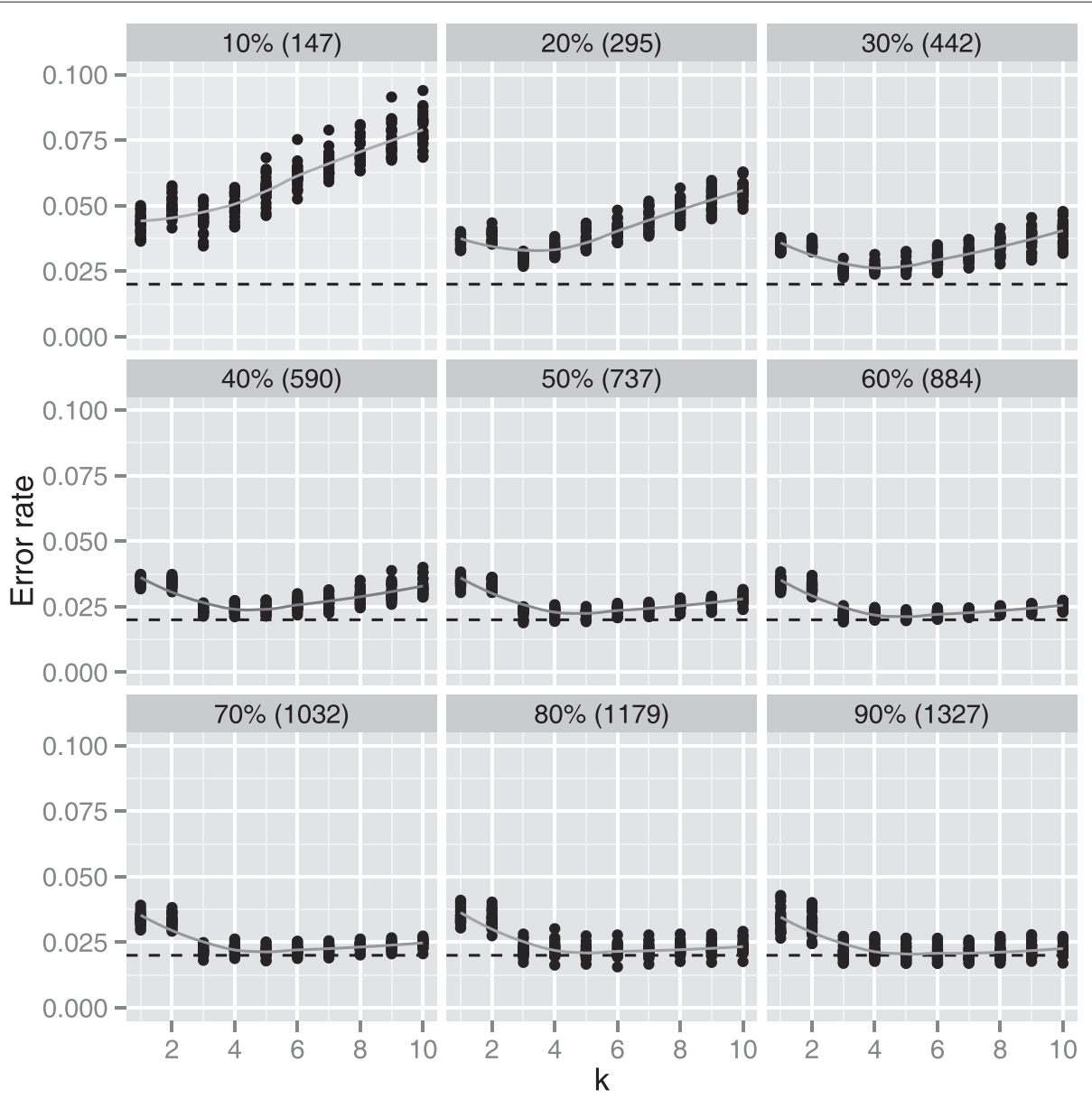

Figure 4 Error rate of k-nearest neighbour prediction from $\boldsymbol{R}$ and $\boldsymbol{\theta}$ of rs 592645 in random subset of samples. Each panel shows the LOOCV error rates of KIR3DL1/3DS1 copy number prediction from $R$ and $\theta$ of rs 592645 in the remaining unlabeled samples when using a different size subset of the training data. The percentage of the complete training data set and the size of the subset is given in the title of each panel. Each point represents the LOOCV error rate averaged over ten multiply imputed qPCR call datasets (using the posterior probabilities from Figure 1). Smoothing lines show the average over 25 independent random subsets of training data. The black dashed line represent the observed error rate in the complete sample. As the size of the training dataset increases the error rate becomes less sensitive to the choice of the parameter $\mathrm{k}$. Only 295 samples are required to achieve LOOCV error rates $<5 \%$ and 590 for error rates $<2.5 \%$.

$\Delta \mathrm{Ct}$, enabled 1474 samples to be confidently assigned to a single copy number group. Over $99 \%$ of all qPCR samples were classified with a posterior probability of copy number group membership greater than 99\%. Further, samples of known copy number, the black points in Additional file 1: Figure S2 corresponding to KIR3DS1-KIR3DL1 copy number 2-1 and 1-2, were assigned to the correct copy number group. We allowed for the limited uncertainty in copy number calling, which mostly stems from distinguishing 2-0 from 3-0 (Figure 1), by means of multiple imputation of ten datasets as described in the Methods.

\section{Imputation into extended samples by integration of SNP and qPCR data}

SNP signals, $R$ and $\theta$, showed strong association with individual copy numbers of KIR3DL1/3SD1 for 19 of 30 SNPs in the KIR3DL1 region (Additional file 1: Table S4). The strongest example is shown for SNP rs592645 in Figure 2, in which clusters can be discerned that correspond closely with qPCR derived $K I R 3 D L 1 / 3 S D 1$ copy numbers.

This figure also illustrates a number of important points regarding using SNP signals for imputation. Firstly, $\theta$ corresponds to the ratio of copies of KIR3DL1 to KIR3DS1, while $R$ corresponds to the total copy number. Secondly, some clusters overlap, particularly along the $R$ axis, making them hard to identify without the qPCR data. Consequently, a large proportion of samples were misclassified when we attempted unsupervised clustering using bivariate finite mixture model approaches, first with PlatinumCNV [18], then with our own, mixture of beta-Gaussian distributions, approach. Finally, the clusters are in slightly different positions in cases and 
controls, reflecting the known sensitivity of genotyping chips to subtle differences in DNA preparation and storage conditions since they were prepared and processed in two different centers $[19,20]$. Instead, we used the qPCR copy numbers as training data to perform supervised classification with knn on the SNP signals, which does not explicitly rely on the identification of distinct clusters.

We first explored the validity of our imputation approach by means of LOOCV in the samples with qPCR data. We examined using all 19 predictive SNPs, or various subsets, and found optimal knn imputation was achieved with the single most predictive SNP, rs592645 (LOOCV rate $=2.0 \%$. By varying the size of the training data, we suggest that only 295 samples are required to achieve LOOCV error rates $<5 \%$ and 590 for error rates $<2.5 \%$ (Figure 4).

\section{No evidence of association of KIR3DL1/3DS1 copy number with T1D}

Finally, we tested for association of KIR3DL1/3DS1 copy number with T1D status. We found no significant evidence of association, in the qPCR data (747 cases and 727 controls), nor in the extended SNP data (6744 cases and 5362 controls), either overall or with any single copy number group (Table 1).

By expanding to these large samples, which would be infeasible to genotype directly with $\mathrm{qPCR}$, we are able to exclude odds ratios outside of the range $[.92 ; 1.08]$ for the common copy number groups with $95 \%$ certainty.

We also repeated the association tests in the subset of individuals, carriers of the HLA-Bw4 epitope, and again detected no significant association (Table 2). A disadvantage of subsetting by HLA-Bw4 is that we lose power by greatly reducing the sample size. A more powerful test

Table 1 Association with T1D tested in the joint copy number group KIR3DS1-KIR3DL1 (a), and in the marginal KIR3DL1 (b) and KIR3DS1 (c) copy number groups

\begin{tabular}{|c|c|c|c|c|c|c|c|c|c|c|}
\hline \multirow{2}{*}{$\begin{array}{l}\text { a) } \\
\text { KIR3DS1-KIR3DL1 }\end{array}$} & \multicolumn{5}{|c|}{ qPCR } & \multicolumn{5}{|c|}{ SNP } \\
\hline & Case:control & Total & OR & $95 \% \mathrm{Cl}$ & p-value & Case:control & Total & OR & $95 \% \mathrm{Cl}$ & p-value \\
\hline $0-2$ & $444: 446$ & 890 & 1.00 & & & 4094:3222 & 7316 & 1 & & \\
\hline $1-1$ & 229:207 & 436 & 1.11 & $0.88-1.40$ & 0.3673 & 2050:1628 & 3678 & 0.99 & $0.92-1.07$ & 0.8349 \\
\hline $2-0$ & $26: 28$ & 54 & 0.92 & $0.52-1.61$ & 0.7713 & $229: 225$ & 454 & 0.79 & $0.65-0.96$ & 0.0193 \\
\hline $2-1$ & $15: 16$ & 31 & 0.94 & $0.46-1.93$ & 0.8695 & 121:101 & 222 & 0.92 & $0.7-1.2$ & 0.5246 \\
\hline $1-2$ & $13: 14$ & 27 & 0.93 & $0.43-2.01$ & 0.8587 & $98: 74$ & 172 & 1.04 & $0.77-1.42$ & 0.7822 \\
\hline $0-1$ & 13:11 & 24 & 1.19 & $0.53-2.68$ & 0.6794 & $116: 77$ & 193 & 1.19 & $0.89-1.59$ & 0.2535 \\
\hline $1-0$ & $4: 3$ & 7 & 1.34 & $0.30-6.02$ & 0.7031 & $25: 21$ & 46 & 0.94 & $0.52-1.68$ & 0.8255 \\
\hline $3-0$ & $3: 2$ & 5 & 1.52 & $0.27-8.62$ & 0.6369 & $11: 14$ & 25 & 0.74 & $0.3-1.82$ & 0.518 \\
\hline Overall & $747: 727$ & 1474 & & & 0.9842 & 6744:5362 & 12106 & & & 0.3552 \\
\hline b) & \multicolumn{5}{|c|}{ qPCR } & \multicolumn{5}{|c|}{ SNP } \\
\hline KIR3DL1 & Case:control & Total & OR & $95 \% \mathrm{Cl}$ & p-value & Case:control & Total & OR & $95 \% \mathrm{Cl}$ & p-value \\
\hline 2 & $457: 460$ & 917 & 1.00 & & & 4192:3296 & 7488 & 1 & & \\
\hline 1 & $257: 234$ & 491 & 1.11 & $0.89-1.38$ & 0.3702 & 2287:1806 & 4093 & 0.99 & $0.92-1.07$ & 0.8883 \\
\hline 0 & $33: 33$ & 66 & 1.01 & $0.61-1.66$ & 0.9795 & $265: 260$ & 525 & 0.8 & $0.67-0.96$ & 0.0151 \\
\hline Overall & $747: 727$ & 1474 & & & 0.6651 & 6744:5362 & 12106 & & & 0.0506 \\
\hline c) & \multicolumn{5}{|c|}{ qPCR } & \multicolumn{5}{|c|}{ SNP } \\
\hline KIR3DS1 & Case:control & Total & OR & $95 \% \mathrm{Cl}$ & p-value & Case:control & Total & OR & $95 \% \mathrm{Cl}$ & p-value \\
\hline 0 & $457: 457$ & 914 & 1.00 & & & 4210:3299 & 7509 & 1 & & \\
\hline 1 & $246: 224$ & 470 & 1.10 & $0.88-1.37$ & 0.4096 & 2173:1723 & 3896 & 0.99 & $0.91-1.07$ & 0.7785 \\
\hline 2 & $41: 44$ & 85 & 0.94 & $0.60-1.47$ & 0.7787 & $350: 326$ & 676 & 0.83 & $0.71-0.97$ & 0.0212 \\
\hline 3 & $3: 2$ & 5 & 1.24 & $0.21-7.28$ & 0.8084 & $11: 14$ & 25 & 0.74 & $0.3-1.82$ & 0.5119 \\
\hline Overall & 747:727 & 1474 & & & 0.8044 & 6744:5362 & 12106 & & & 0.1494 \\
\hline
\end{tabular}

No evidence of a significant, joint or marginal, effect was detected in the qPCR dataset, 747 cases and 727 controls, nor in the SNP dataset, 6744 cases and 5362 controls. Case-control counts shown are derived from the most likely copy number assignment across the ten multiply imputed qPCR and SNP datasets. Statistical inference for association is derived from the multiply imputed datasets using the R mitools package [13]. The last row of each table contains the pooled $p$-value for each association test using the $\mathrm{R}$ mice package [14]. 
for interaction between unlinked genes is a case-only test [16]. If there were an interaction between KIR3DL1/3DS1 and HLA-Bw4 then this should be detectable as a difference in $K I R 3 D L 1 / 3 D S 1$ copy number frequencies across HLA-Bw4 strata in the cases. However, we found no significant evidence for association in either the qPCR or SNP data sets, before or after summarising the KIR copy number by presence/absence to increase power by reducing the degrees of freedom (Table 3 ).

\section{Conclusion}

Regions with great allelic and copy number variation are difficult to properly assess using GWAS. While genomewide SNP arrays are typically cost effective ways to assay common genetic variation, very polymorphic regions can make the design of SNP probes that bind uniquely to their target region, difficult or impossible. This has resulted in low SNP coverage in the KIR region for the common SNP arrays. The SNPs that do exist on arrays are often discarded during the QC phase of any GWAS because they do not exhibit the expected three clusters. On the other hand, assaying individual genes can prove expensive. For example, the qPCR assays used here to target KIR3DL1 and KIR3DS1 cost $£ 12$ per sample.

Further, qPCR derived data, despite careful design and multiplexing, remain subject to noise (plate 22 Additional file 1: Figure S1). We ameliorated this through QC and normalisation across plates, and then jointly clustering KIR3DL1 and KIR3DS1, to exploit the correlation between the $\Delta \mathrm{Ct}$ values. The advantage of joint clustering is demonstrated in qPCR plate 10, where noisy cases (Additional file 1: Figure S1.f) are difficult to assign as one or two copies based solely on their KIR3DL1 $\Delta \mathrm{Ct}$, but are much more clearly distinguishable when we also consider their KIR3DS1 $\triangle \mathrm{Ct}$ value (Additional file 1: Figure S2).

Table 2 Association with T1D conditional on the presence of the respective HLA-Bw4 epitope, tested in the joint copy number group KIR3DS1-KIR3DL1 (a), and in the marginal KIR3DL1 (b) and KIR3DS1 (c) copy number groups

\begin{tabular}{|c|c|c|c|c|c|c|c|c|c|c|}
\hline \multirow{2}{*}{$\begin{array}{l}\text { a) HLA-Bw4 subset } \\
\text { KIR3DS1-KIR3DL1 }\end{array}$} & \multicolumn{5}{|c|}{ qPCR } & \multicolumn{5}{|c|}{ SNP } \\
\hline & Case:control & Total & OR & $95 \% \mathrm{Cl}$ & p-value & Case:control & Total & OR & $95 \% \mathrm{Cl}$ & $\mathrm{p}$-value \\
\hline $0-2$ & $259: 286$ & 545 & 1.00 & & & 1027:1157 & 2184 & 1 & & \\
\hline $1-1$ & $123: 128$ & 251 & 1.06 & $0.79-1.43$ & 0.6976 & $555: 582$ & 1137 & 1.08 & $0.93-1.24$ & 0.3119 \\
\hline $2-0$ & $16: 15$ & 31 & 1.22 & $0.58-2.57$ & 0.5985 & $59: 88$ & 147 & 0.76 & $0.54-1.07$ & 0.1133 \\
\hline $2-1$ & $7: 13$ & 20 & 0.59 & $0.23-1.51$ & 0.2754 & $34: 40$ & 74 & 0.93 & $0.58-1.48$ & 0.7529 \\
\hline $1-2$ & $8: 8$ & 16 & 1.10 & $0.41-2.98$ & 0.8450 & $24: 33$ & 57 & 0.85 & $0.5-1.45$ & 0.5502 \\
\hline $0-1$ & $10: 7$ & 17 & 1.58 & $0.59-4.20$ & 0.3621 & $36: 24$ & 60 & 1.69 & $1-2.85$ & 0.0491 \\
\hline $1-0$ & $2: 1$ & 3 & 2.21 & $0.20-24.50$ & 0.5187 & $7: 4$ & 11 & 1.97 & $0.58-6.76$ & 0.2793 \\
\hline $3-0$ & $3: 0$ & 3 & & & & $5: 0$ & 5 & & & \\
\hline Overall & $428: 458$ & 886 & & & 0.8978 & 1747:1928 & 3675 & & & 0.2173 \\
\hline b) HLA-Bw4 subset & \multicolumn{5}{|c|}{ qPCR } & \multicolumn{5}{|c|}{ SNP } \\
\hline KIR3DL1 & Case:control & Total & OR & $95 \% \mathrm{Cl}$ & p-value & Case:control & Total & OR & $95 \% \mathrm{Cl}$ & p-value \\
\hline 2 & 267:294 & 561 & 1.00 & & & 1051:1190 & 2241 & 1 & & \\
\hline 1 & $140: 148$ & 288 & 1.04 & $0.78-1.38$ & 0.7787 & $625: 646$ & 1271 & 1.09 & $0.95-1.26$ & 0.1975 \\
\hline 0 & $21: 16$ & 37 & 1.45 & $0.74-2.83$ & 0.2822 & $71: 92$ & 163 & 0.88 & $0.64-1.21$ & 0.4181 \\
\hline Overall & $428: 458$ & 886 & & & 0.5563 & 1747:1928 & 3675 & & & 0.2586 \\
\hline c) HLA-Bw4-80I subset & \multicolumn{5}{|c|}{ qPCR } & \multicolumn{5}{|c|}{ SNP } \\
\hline KIR3DS1 & Case:control & Total & OR & $95 \% \mathrm{Cl}$ & p-value & Case:control & Total & OR & $95 \% \mathrm{Cl}$ & p-value \\
\hline 0 & 159:187 & 346 & 1.00 & & & $649: 733$ & 1382 & 1 & & \\
\hline 1 & $93: 83$ & 176 & 1.32 & $0.92-1.90$ & 0.1370 & $383: 366$ & 749 & 1.18 & $0.99-1.41$ & 0.0628 \\
\hline 2 & $12: 14$ & 26 & 1.01 & $0.45-2.24$ & 0.9842 & $61: 75$ & 136 & 0.91 & $0.64-1.3$ & 0.607 \\
\hline 3 & $2: 0$ & 2 & & & & $3: 0$ & 3 & & & \\
\hline Overall & $266: 284$ & 550 & & & 0.5209 & 1096:1174 & 2270 & & & 0.2416 \\
\hline
\end{tabular}

Association is tested in the subset of individuals carriers of an HLA-Bw4 epitope for the joint KIR3DS1-KIR3DL 1 (a) and marginal KIR3DL 1 (b) copy number groups and also tested in the subset of individuals carriers of the HLA-Bw4-80I epitope for the marginal KIR3DS1 (c) copy number group. Case-control counts shown are derived from the most likely copy number assignment across the ten multiply imputed qPCR and SNP datasets. Statistical inference for association is derived from the multiply imputed datasets using the R mitools package [13]. The last row of each table contains the pooled p-value for each association test using the $\mathrm{R}$ mice package [14]. 
Table 3 Case-only $\chi^{2}$ test for interaction between KIR3DS1-KIR3DL1 and HLA-Bw4, across the ten multiply imputed qPCR and SNP datasets

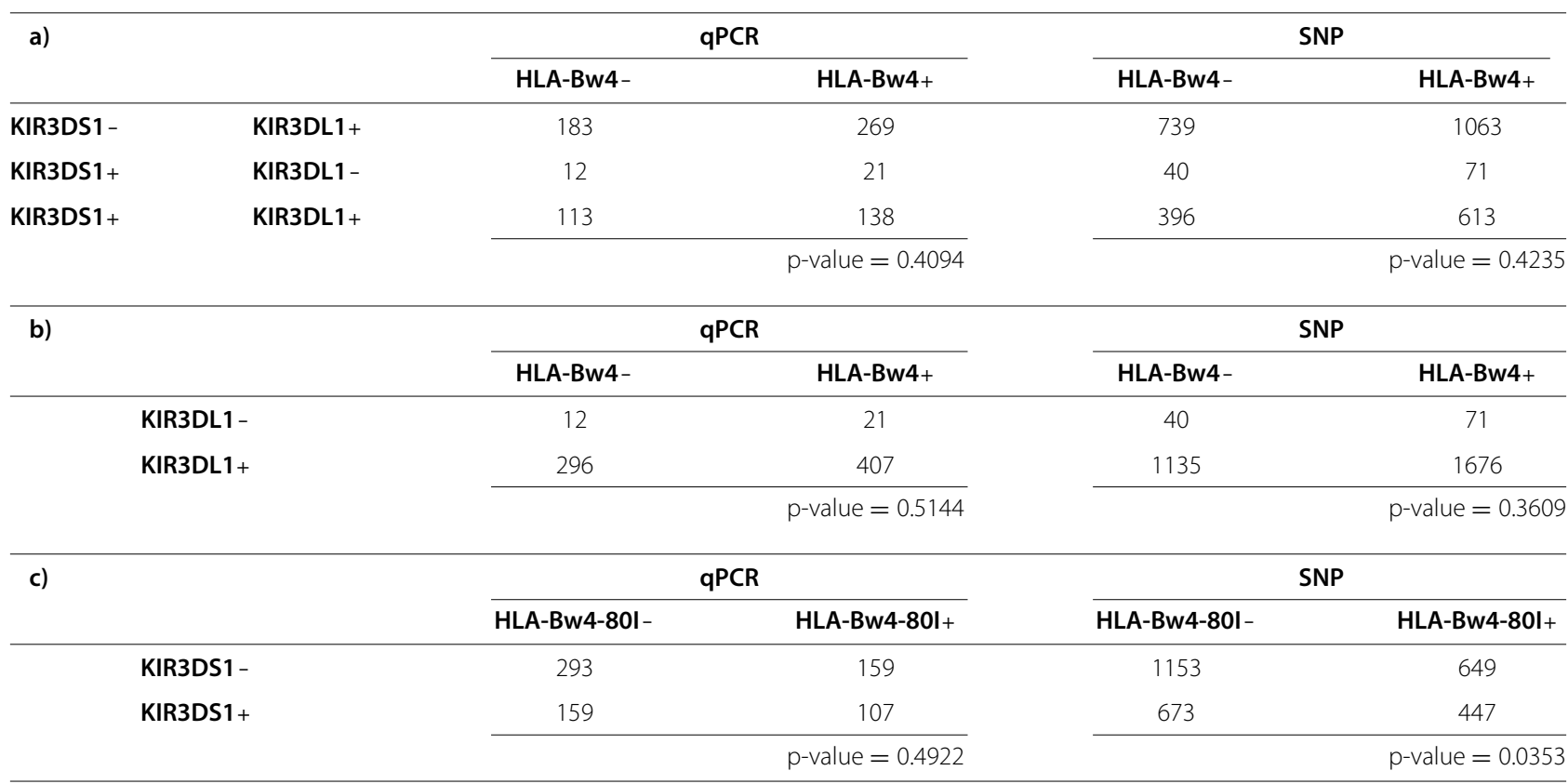

Counts in each contingency table are derived from the most likely copy number assignment across the multiply imputed datasets. To reduce the degrees of freedom and improve power, we summarise copy numbers higher or equal to one by presence $(+)$ and zero by absence (-). The pooled $p$-value of each $\chi^{2}$ test, across the multiply imputed datasets, is given in the last row of each contingency table. We find no significant association with HLA-Bw4, within cases, in either the joint (a) or the marginal (b)(c) KIR3DS1-KIR3DL1 distributions.

As receptors for HLA class I molecules, KIR genes are important candidates for T1D and other diseases that associate with HLA variation. However, researchers have been unable to fully assess their candidacy due to lack of coverage in GWAS and the complexity and expense of KIR gene-specific assays. So far, KIR association studies for T1D have involved small samples sizes and have probed for presence/absence of multiple KIR genes whilst ignoring the respective copy numbers of these genes, with mixed results and no consistent pattern of association emerging. However, copy number variation in KIR could be important, as it is a mechanism which gives rise to a large diversity of haplotypes [1]. Our hybrid approach, as outlined in Additional file 1: Figure S4, allowed us to perform the (twenty-fold) largest study of KIR3DL1/3DS1 copy number in T1D to date, and to test for association in eight of the most frequent copy number groups (Additional file 1: Table S5). In 12106 samples, we found no association of KIR3DL1/3DS1 copy number with T1D, alone or conditional on presence of the HLA-Bw4 epitope. Our results suggest that, despite the association of certain HLA-A and HLA-B alleles with T1D and the established biological interaction between HLA-Bw4 and KIR3DL1, copy number variation in KIR3DL1/3DS1 is unlikely to have a significant effect on the risk of developing T1D. Other KIR genes that are in high linkage disequilibrium with KIR3DL1 and KIR3DS1 are also unlikely to be associated. According to the Allele Frequency Net database [21], these include KIR2DS4 (97\%) and KIR2DL3 (86\%), for KIR3DL1 and, KIR2DL5 (81\%), KIR2DS5 (84\%) and KIR2DS1 (92\%), for KIR3DS1 (http://www.allelefrequencies.net/kir6010a. asp). Thus, copy number variation in KIR3DL1/3DS1 or neighbouring genes is unlikely to be an important risk factor in T1D.

In order to better understand why rs592645 is the best SNP for predicting copy number variation in KIR3DL1/3DS1, we used BLAT [22] to match the probe sequences of rs592645 on ImmunoChip against the allelic sequences of all KIR genes available from the Immuno Polymorphism Database [23]. Interestingly, we found that the SNP probes do not target KIR3DL1/3DS1 but instead bind uniquely to the fifth intron of KIR2DL4, a neighbouring framework gene. Examining the KIR2DL4 alleles matched by the rs592645 probes, we discovered that the SNP probes are in fact picking up copy number variation of KIR2DL4*005, an allele of KIR2DL4 that undergoes copy number variation along with KIR3DL1/3DS1 [24]. This explains the small but persistent misclassification error rate of $2 \%$ since our imputation is based on linkage disequilibrium between rs592645 and KIR3DL1/3DS1 rather than on perfect discrimination between our target genes. We have identified 27 samples, which we believe, are consistently misclassified due to imperfect linkage disequilibrium (Additional file 1: Table S6). 
We have observed other SNPs with more than three clusters that may correlate with copy number of other KIR genes and, given the availability of qPCR results, could be imputable in a similar manner.

\section{Additional file}

Additional file 1: Supplementary figures and tables.

\section{Abbreviations}

CNV: Copy number variation; HLA: Human Leukocyte Antigen; KIR: Killer Immunoglobulin-like Receptor; knn: k-nearest neighbour; LOOCV: Leave-one-out cross-validation; qPCR: Quantitative polymerase chain reaction; SNP: Single nucleotide polymorphism; T1D: Type 1 diabetes.

Competing interests

The authors declare that they have no competing interests.

\section{Authors' contributions}

Conceived and designed the experiments: JA Todd. Designed the GPCR assays: JJ, WJ, JA Traherne and JT. Performed the qPCR experiments for KIR3DL1/3DS1: DJS. Performed ImmunoChip genotyping: SO-G, W-MC, PC and SSR. Prepared data: HS, NMW, HG and OSB. Statistical analysis: NP, CW, JMMH and HS. Wrote the manuscript: NP and CW. Reviewed and revised the manuscript: JMMH, DS, JA Traherne, NMW and JA Todd. All authors have read and approved the manuscript.

\section{Acknowledgements}

This work was funded by the JDRF, the Wellcome Trust (WT grants 061858 and 091157) and the National Institute for Health Research (NIHR) Cambridge Biomedical Research Centre. The research leading to these results has received funding from the European Union's 7th Framework Programme (FP7/2007-2013) under grant agreement no.241447 (NAIMIT). We thank David Dunger, Barry Widmer, and the British Society for Paediatric Endocrinology and Diabetes for the TID case collection. We would like to thank the UK Medical Research Council and Wellcome Trust for funding the collection of DNA for the British 1958 Birth Cohort (MRC grant G0000934 and WT grant 068545/Z/02). DNA control samples were prepared and provided by S. Ring, R. Jones, M. Pembrey, W. McArdle, D. Strachan and P. Burton. NP is supported by the Medical Research Council Doctoral Training Grant (C006148). CW and HG are funded by the Wellcome Trust (WT grant 089989). JT and JAT are supported by the Medical Research Council (MRC Grant G0901682) and the Wellcome Trust (WT 08982/Z/09/Z). The Cambridge Institute for Medical Research (CIMR) is in receipt of a Wellcome Trust Strategic Award (100140).

\section{Author details}

1 JDRF/Wellcome Trust Diabetes and Inflammation Laboratory,

Cambridge Institute for Medical Research, University of Cambridge, Wellcome Trust/MRC Building, CB2 OXY, Cambridge, UK. ${ }^{2}$ Cardiovascular Epidemiology Unit, Department of Public Health and Primary Care, University of Cambridge, Strangeways Research Laboratory, CB1 8RN, Cambridge, UK. ${ }^{3}$ Center for Public Health Genomics, University of Virginia, 22908-0717, Charlottesville, Virginia, USA. ${ }^{4}$ University of Florida Genetics Institute, 32610-3610, Gainesville, Florida, USA. ${ }^{5}$ Division of Immunology, Department of Pathology, University of Cambridge, Tennis Court Road, CB2 1QP, Cambridge, UK. ${ }^{6}$ Cambridge Institute for Medical Research, University of Cambridge, Wellcome Trust/MRC Building, CB2 OXY, Cambridge, UK. ${ }^{7}$ MRC Biostatistics Unit, Institute of Public Health, University Forvie Site, Robinson Way, CB2 OSR, Cambridge, UK.

Received: 17 December 2013 Accepted: 3 April 2014 Published: 11 April 2014

\section{References}

1. Jiang W, Johnson C, Jayaraman J, Simecek N, Noble J, Moffatt MF, Cookson WO, Trowsdale J, Traherne JA: Copy number variation leads to considerable diversity for B but not A haplotypes of the human KIR genes encoding NK cell receptors. Genome Res 2012, 22(10):1845-1854.
2. Gumperz JE, Barber LD, Valiante NM, Percival L, Phillips JH, Lanier LL, Parham P: Conserved and variable residues within the Bw4 motif of HLA-B make separable contributions to recognition by the NKB1 killer cell-inhibitory receptor. J Immunol 1997, 158(11):5237-5241.

3. Vivian JP, Duncan RC, Berry R, O'Connor GM, Reid HH, Beddoe T, Gras S, Saunders PM, Olshina MA, Widjaja JML, Harpur CM, Lin J, Maloveste SM, Price DA, Lafont BAP, McVicar DW, Clements CS, Brooks AG, Rossjohn J: Killer cell immunoglobulin-like receptor 3DL1-mediated recognition of human leukocyte antigen B. Nature 2011, 479(7373):401-405.

4. Martin MP, Qi Y, Gao X, Yamada E, Martin JN, Pereyra F, Colombo S, Brown EE, Shupert WL, Phair J, Goedert JJ, Buchbinder S, Kirk GD, Telenti A, Connors M, O'Brien SJ, Walker BD, Parham P, Deeks SG, McVicar DW, Carrington M: Innate partnership of HLA-B and KIR3DL1 subtypes against HIV-1. Nat Genet 2007, 39(6):733-740.

5. Martin MP, Gao X, Lee JH, Nelson GW, Detels R, Goedert JJ, Buchbinder S, Hoots K, Vlahov D, Trowsdale J, Wilson M, O'Brien SJ, Carrington M: Epistatic interaction between KIR3DS1 and HLA-B delays the progression to AIDS. Nat Genet 2002, 31(4):429-434.

6. Nejentsev S, Howson JMM, Walker NM, Szeszko J, Field SF, Stevens HE, Reynolds P, Hardy M, King E, Masters J, Hulme J, Maier LM, Smyth D, Bailey R, Cooper JD, Ribas G, Campbell RD, Clayton DG, Todd JA: Wellcome Trust Case Control Consortium: Localization of type 1 diabetes susceptibility to the MHC class I genes HLA-B and HLA-A. Nature 2007 450(7171):887-892.

7. Howson JMM, Walker NM, Clayton D, Todd JA: Diabetes Genetics Consortium: Confirmation of HLA class II independent type 1 diabetes associations in the major histocompatibility complex including HLA-B and HLA-A. Diabetes Obes Metab 2009,

11:31-45.

8. Mehers KL, Long AE, van der Slik AR, Aitken RJ, Nathwani V, Wong FS, Bain S, Gill G, Roep BO, Bingley PJ, Gillespie KM: An increased frequency of NK cell receptor and HLA-C group 1 combinations in early-onset type 1 diabetes. Diabetologia 2011, 54(12):3062-3070.

9. Barrett JC, Clayton DG, Concannon P, Akolkar B, Cooper JD, Erlich HA, Julier C, Morahan G, Nerup J, Nierras C, Plagnol V, Pociot F, Schuilenburg H, Smyth DJ, Stevens H, Todd JA, Walker NM, Rich SS: Genome-wide association study and meta-analysis find that over 40 loci affect risk of type 1 diabetes. Nat Genet 2009, 41(6):703-707.

10. Nikula T, West A, Katajamaa M, Lonnberg T, Sara R, Aittokallio T, Nevalainen O, Lahesmaa R: A human ImmunoChip cDNA microarray provides a comprehensive tool to study immune responses. $J$ Immunol Methods 2005, 303(1-2):122-134.

11. Young D, Hunter D, Chauveau D, Benaglia T: mixtools: an R package for analyzing finite mixture models. J Stat Software 2009, 32(6):1-29. [http://www.jstatsoft.org/v32/i06/]

12. Little RJ, Rubin DB: Statistical Analysis With Missing Data. Wiley; 1987.

13. Lumley T: mitools: Tools for Multiple Imputation of Missing Data; 2012. [http://CRAN.R-project.org/package=mitools]. [R package version 2.1]

14. van Buuren S, Groothuis-Oudshoorn K: mice: Multivariate Imputation by Chained Equations in R. J Stat Software 2011, 45(3):1-67. [http://www.jstatsoft.org/v45/i03/]

15. Cordell $\mathrm{HJ}$ : Estimation and testing of genotype and haplotype effects in case-control studies: comparison of weighted regression and multiple imputation procedures. Genet Epidemiol 2006, 30(3):259-275.

16. Yang Q, Khoury MJ, Sun F, Flanders WD: Case-only design to measure gene-gene interaction. Epidemiology 1999, 10(2):167-170

17. Cordell $\mathrm{HJ}$ : Detecting gene-gene interactions that underlie human diseases. Nat Rev Genet 2009, 10(6):392-404.

18. Kumasaka N, Fujisawa H, Hosono N, Okada Y, Takahashi A, Nakamura Y, Kubo M, Kamatani N: PlatinumCNV: a Bayesian Gaussian mixture model for genotyping copy number polymorphisms using SNP array signal intensity data. Genet Epidemiol 2011, 35(8):831-844.

19. Clayton DG, Walker NM, Smyth DJ, Pask R, Cooper JD, Maier LM, Smink LJ, Lam AC, Ovington NR, Stevens HE, Nutland S, Howson JMM, Faham M, Moorhead M, Jones HB, Falkowski M, Hardenbol P, Willis TD, Todd JA Population structure, differential bias and genomic control in a large-scale, case-control association study. Nat Genet 2005 37(11):1243-1246

20. Plagnol V, Cooper JD, Todd JA, Clayton DG: A method to address differential bias in genotyping in large-scale association studies. PLoS Genet 2007, 3(5):e74. 
21. Gonzalez-Galarza FF, Christmas S, Middleton D, Jones AR: Allele frequency net: a database and online repository for immune gene frequencies in worldwide populations. Nucleic Acids Res 2011, 39(Database issue):D913-D919.

22. Kent WJ: BLAT-the BLAST-like alignment tool. Genome Res 2002, 12(4):656-664.

23. Robinson J, Mistry K, McWilliam H, Lopez R, Marsh SGE: IPD-the Immuno Polymorphism Database. Nucleic Acids Res 2010, 38(Database issue):D863-D869.

24. Gómez-Lozano N, Estefanía E, Williams F, Halfpenny I, Middleton D, Solís $R$, Vilches $C$ : The silent KIR3DP1 gene (CD158c) is transcribed and might encode a secreted receptor in a minority of humans, in whom the KIR3DP1, KIR2DL4 and KIR3DL1/KIR3DS1 genes are duplicated. Eur J Immunol 2005, 35:16-24.

doi:10.1186/1471-2164-15-274

Cite this article as: Pontikos et al:: A hybrid qPCR/SNP array approach allows cost efficient assessment of KIR gene copy numbers in large samples. BMC Genomics 2014 15:274.

\section{Submit your next manuscript to BioMed Central} and take full advantage of:

- Convenient online submission

- Thorough peer review

- No space constraints or color figure charges

- Immediate publication on acceptance

- Inclusion in PubMed, CAS, Scopus and Google Scholar

- Research which is freely available for redistribution

Submit your manuscript at www.biomedcentral.com/submit
( Biomed Central 